\title{
Research of the Erhu Teaching at Comprehensive Universities
}

\author{
Saijun Chen \\ School of Art, Yangtze University, Jingzhou, China, 434023 \\ qubaolong_78@163.com
}

\begin{abstract}
Keywords: Erhu professional teaching; Erhu playing; The art of music performance; Comprehensive universities
\end{abstract}

\begin{abstract}
This article takes the Erhu performance professional teaching as its research object and the music department of School of Arts, Yangtze University as its research background. By tackling with two aspects, the transformation of the Erhu teaching concepts and the reform of Erhu teaching method, this article studies the research achievements of those Erhu educational scholars, analyzes the problems existing in the Erhu professional teaching at comprehensive universities and gives some possible explanation and reasons why the teaching objectives deviate. This article also points out the differences between the universal education at the comprehensive universities and the 'elite education' from conservatories and the 'teacher education' from teachers' colleges and universities by expounding the properties of the school and the training objectives. Finally this article poses several suggestions to improve and reform the current Erhu professional teaching situation at comprehensive universities. The Erhu professional teaching at comprehensive universities has long been ignored by the experts and scholars. The research shown in this article has significant meaning in this field, providing reference for the research and development of Erhu professional teaching at comprehensive universities. It is also expected to be useful and applicable for the Erhu professional teaching at comprehensive universities in the future.
\end{abstract}

\section{Introduction}

Since 1922, the year Mr. Liu Tianhua was hired as an Erhu teacher by the Training Institute of Music of Peking University, Erhu has started the process of transforming from the "civilian state" to the professional state. Especially after the founding of new China, the national professional art colleges and universities through the transformation and new construction, formed the national professional music education. Some places of education and academic research started to take shape and thus provided the professional development of Erhu In recent years, with the continuous development of higher education, many universities have set up art departments and Erhu has become one of the most common instrumental music courses at universities. In the face of "elite" training mode of music colleges and universities, as well as the Teachers' College's "to cultivate qualified teachers" education goals, comprehensive university's Erhu professional teaching is worthy of further research on how to develop in such dilemma, how to ensure the teaching quality and talent training specifications, how to adapt to the needs of society, and how to rush out an effective way of teaching.

Based on the contemporary Erhu scholars' researching on Erhu teaching at conservatories and Teacher's College, taking the Erhu professional teaching at comprehensive universities as the starting point, drawing lessons from other related successful excellent teaching achievements, integrating with the author's more than ten years' Erhu teaching experience, this article systematically researches on the Erhu teaching characteristics at comprehensive universities, put forward the practical reform measures in view of the current problems existing in the Erhu teaching. Hopefully, it will draw public attention from Erhu teaching experts and scholars, and thus further improve the level of Erhu professional teaching at comprehensive universities, cultivating for the country innovative talents who have both solid theoretical foundation and practical ability 


\section{The Present Situation of Erhu Teaching at Comprehensive Universities}

With the in-depth development of China's higher education reform, many colleges and universities are amalgamated or upgraded to comprehensive universalities on the original foundation and have added music discipline to the universities. This action has added a high and historic note for our country's higher music education and has had significant and far-reaching impact on our country's music culture construction and the promotion of cultural and ideological progress.

However, in recent years, phenomenon of "students are not happy to learn, teachers are not good at teaching" emerged at comprehensive universities. On one hand, it attributed to the fact that a comprehensive university discipline construction imbalance leads to the weakness of the academic research of the teaching environment. On the other hand, the Erhu teaching courses at comprehensive universities cover a wide range and the students are varied at the professional level, which lead to the problem of the lack of scientific management and systematization in the professional curriculum setting, teaching methods, teaching contents and teaching materials

Folk Music Teaching is not Valued. At the comprehensive universities, the dominant disciplines are usually the science, the technology, the literature and the history. Music teaching, an auxiliary class discipline to complete the school discipline, is regarded less important than other subjects. Firstly, comprehensive universities often take music teaching as nonessential and thus have fewer teaching investment on it. The teaching equipments are not well equipped or completed. Secondly, there is strong imbalance between the discipline construction and the teachers distribution due to the disparity proportion between the students who major in vocal music and musicology the students who major in folk music. Folk music teaching is marginalized and Erhu professional teaching is lowered and not valued.

Lack of Good Teaching Atmosphere. It is the discipline construction imbalance of folk music teaching at comprehensive universities that makes the folk music teachers have doubt about their value of the major and sense of identity. Consequently, they gradually lose enthusiasm in teaching and academic research, which to a large extent affect the improvement of teaching quality. On the other hand, compared with professional music colleges and universities, the comprehensive universities have no advantages on the student's source. The students' admission standards are low and the standards, coupled with the national enrollment expansion of colleges, are lowered again and again. All these situations will lead to a very terrible vicious circle, making comprehensive universities Erhu education levels come to a standstill for a long time.

Since 1922, when Mr. Liu Tianhua made reforms on the traditional Erhu, all conservatories over the country had applied the Liu Erhu teaching method. Erhu art has boomed all over the country, the status of the Erhu has also risen sharply. As written by his brother Liu Bannong in his book on his late brother, "Erhu is humble but is able to be a choice product because of its talent. Erhu arts cannot reach its status in the history of China folk music development without the most valuable help from Mr. Liu Tianhua. Liu Erhu teaching method has contributed a lot to it.

Under such circumstances, the Erhu teaching of comprehensive universities have imitated blindly each music teaching mode of the conservatories, applying "liu" Erhu teaching method into the Erhu class. But this blind and mechanical application is not completely suitable for comprehensive university environment. For example, in the past, there are certain reasons why comprehensive universities imitate the conservatoires' one to one teaching mode. It is mainly in order to strengthen the students' professional foundation and to improve students' level of Erhu playing. However, in recent years, because of the country's vigorous popularization of national cultural quality, and the enrollment expansion of comprehensive universities, the number of students enrolled is increasing year by year. So, the strengthening of the comprehensive university excessive professional teaching and the blind pursuit of elite education cannot meet the popular social demand and are not in conformity with the national popularization of art education. To change this situation, Erhu group lessons appear. Three or five students are taught together at the same time, the so-called "one too many". But the students' Erhu playing abilities vary and it's hard for three or five students to "go 
together". While group lessons solved the problems of the lack of teaching staff and the task of the national popularization of art education, it has the problem too. It is hard to teach students in accordance with their aptitude and is only "a handful of rice". This is confusion.

Anyway, because the Erhu major has been integrated into the comprehensive universities dominated by the disciplines of the science, the technology, the literature and the history, and it has been affected by college enrollment expansion and influenced by the traditional teaching mode of the conservatories, the comprehensive universities have many problems on disciplines development, students' level and teaching modes. These problems go against the development of Erhu teaching at comprehensive universities, and do no good to cultivate Erhu professional talents with comprehensive quality.

\section{Measures to Improve Comprehensive University Erhu Professional Teaching Quality}

Due to the particularity of comprehensive university music discipline orientation, comprehensive university of Erhu teaching can no longer continue to adopt the traditional teaching mode of the conservatories. The traditional Erhu teaching mode should have an all-round reformation to meet the talent training objects and different teaching methods should be applied according to the different levels of students, thus improving the quality of Erhu teaching.

Grasp the Teaching Principle of Teaching According to Student's Aptitude. According to their Erhu performing abilities, students at the comprehensive universities can be roughly divided into two categories: one is the artificial type. These students had relatively professional training before enrolled into universities and had mastered the basic playing methods. They improved their performance in order to meet the standards of the College Entrance Examination. Although they could pay some difficult pieces of music, they play rough and have many problems on intonation, rhythm, timbre, and etc. The other one is the short term speeding form. These students have not standard professional training before enrolled and even have problems on basic playing method such as how to hold the musical instrument in a proper way. Although they could play some examination-oriented pieces of music, they are not capable of giving performance.

For the first kind of students, after knowing their actual ability, the teacher can base the professional training on their actual ability and have specific training to improve their weak parts. At the same time, the teacher should complete other missing basic parts. On one hand, it is essential to strengthen the practice skills to have further solid foundation; on the other hand, it can also carry out the guide to music performance, in order to realize the overall ascension of professional power.For the second category of students, teachers need to have enough patience in teaching, and must teach from the most basic playing method, regulating the players' playing position, correcting their intonation and rhythm, striving to help students establish correct performing method as soon as possible.

For these two types of students, the teacher should hold rational, objective teaching ideas, and avoid by all means negating what the students had learned before and avoid starting from the beginning. The teacher should give standard training and focus on adjusting, thus improving and fulfilling the students' basic skills. Total rejection teaching method would definitely have negative influence on students' learning enthusiasm.

Choose the Reasonable Teaching Materials. Today the kinds of Erhu teaching materials can be roughly divided into tutorial class, music class, practice class, and test class. Since the $1990 \mathrm{~s}$, the Erhu construction of teaching material boomed into full flowering stage, textbooks of the different degree levels, different types, different styles, written collectively or individually, emerging like mushrooms. There are more than one hundred teaching materials available on the market. Erhu teaching textbooks are developing diversity [2]. This also creates a difficult problem for the comprehensive university on how to choose suitable teaching material for students at different level to make reasonable and effective teaching plan. Standard and scientific textbooks can correctly guide students on idea learning, the purpose of the study and learning goals [3]. Comprehensive universities have no scientific and systematic teaching materials, leading to the weak performance by those Erhu 
major students. Therefore, for the basic teaching, Erhu teacher should pay attention to choose appropriate teaching material and take the principles of "targeting" and "periodicity".

For example, for the Erhu performing professional freshmen and sophomores, students can use the textbook "Erhu primer" by Zhao Han yang [4], the "Erhu systematic advance gradus" by Liu Changfu [5] (beginning, intermediate section), "the Erhu scales" by Wang guotong [6] (beginning, intermediate section), "the Erhu's three hundred basic exercises" co-authored by Wang guotong and Zhao Han yang [7], " Erhu bowing practice law 37 pieces" by Yan Jiemin [8], the" Erhu mini practice pieces" by Zhao Han yang [9], etc.

The students of this phase should give priority to master correct, standard methods of playing, should pay attention to the decomposition of monomial skill training content, and should combine with strong melody etudes to arouse the students' interest in learning. For Erhu performing professional juniors and seniors, students can choose the style of Erhu gradus by Xue Ke[10], "the 158 Erhu style practice pieces" co-authored by Wang guotong and Zhao Han yang [11], the "Erhu etudes fluid 26" by Su Hanxing [12], etc. The students of this phase has already mastered some playing techniques and some music theory basis, so in terms of choice of content they should pay attention to the training of composite techniques, focusing on the mastery of style technique.

To Strengthen the Teaching of Professional Theoretical Knowledge. Under the current Erhu teaching content of the comprehensive universities, the emphasis is put on the improvement of Erhu playing skills and the number increase on the tracks. Little is explained on the composing background of the Erhu works, characteristics of the styles and the development of Erhu which is relied on the ethnic folk music and culture. Most of the students' performance is the imitations of the teachers'. It lacks of personal understanding of the work, the understanding of Erhu as a muscial instrument, and the understanding of traditional music culture. Comprehensive universities aim to cultivate students as not only social music educators, but also the disseminator of traditional Erhu music, inter-disciplinary talents who can play as well as teach others how to play and how to interpret the connotation. Therefore, the teacher should not ignore imparting professional theoretical knowledge while teaching the performance skills.

In Erhu learning process, students' ability to grasp the play style of the Erhu works is bad. For the university students who have the independent thinking ability, it is necessary to put the abstract perceptual learning on a rise to a height of theory. The teacher can give explanation on the Erhu playing theory in the class, let the students to know "what" and to "know why, inspire them to think rationally and solve the hurdles faced in the practice in the future, and eventually help students have performance skills breakthrough. Only after the students have such theoretical basis, can they give precise interpretation of Erhu playing technology in the later Erhu teaching positions. In addition, basic theoretical knowledge introduction to the Chinese national instrumental music art appreciation can be introduced to students. Erhu development history, introductions to the Erhu art schools, they styles of the famous musician and pieces of music should also be included.

Actively Carry out Professional Demonstration and Seminar. In order to enhance the students' professional knowledge and theory, teachers can regularly organizes the student to give a professional demonstration at a small range. It is required that every student plays a complete works and a subsequent appraisal meeting will be organized. Teachers will give comments and opinion and students share good playing experience and methods. Such method can stimulate the initiative of the students' autonomous learning, and the exchange of experience among the students can cause the resonance of the students, and promote the students to actively think about professional questions: "Is this method feasible for me?", "how can I play better?" At the same time it also can give the students more opportunities to practice, thus improving their performance.

In addition, the teacher can also regularly organize Erhu lecture, give effective introduction and summary about Erhu playing skills, playing theory, and practice methods, and guide students to study more efficiently. At the same time, it is essential to take advantage of the complete discipline of the comprehensive universities, strengthen the study of multidisciplinary theory knowledge, and improve 
the students in the theory of history, literature, drama and etc. to cultivate versatile and applicable talents who have strong theory background and excellent playing skills.

Create Stage Opportunities for Students. As the saying goes, "ten minutes on stage, ten years of hard work behind the stage". Even students have "ten years hard work behind the stage", if they have no opportunity to perform and they have no confidence on the stage performance, ten minutes on stage is not necessarily a success. .In order to develop students' good psychological quality, schools should create as many performance opportunities as possible, such as, the regular students practice concert, special concert, university-enterprise cooperation performance, and etc. Stage practice can make students not only master skills of acting as early as possible, strengthen the psychological quality, but can also improve students' ability to play. But one thing is important to note, that is, university-enterprise cooperation performance should be taken properly and orderly, and make the classroom teaching and stage practice supplement each other.

Comprehensive universities target to cultivate comprehensive talents with higher cultural quality, strong artistic expression ability and comprehensive practice ability. Therefore, it is necessary to take into consideration of the different levels of the students at the comprehensive universities, understand students' theoretic and playing ability and have targeted teaching. To enhance the teaching effect and improve the classroom efficiency, an open teaching environment should be encouraged. We should give the fullest play to the students' subjective initiative and take advantage of the current teaching method to improve, develop and bring forth more methods to promote students' learning, and to create a better and virtuous circle of teaching mechanism.

\section{Conclusion}

Erhu teaching and researching at comprehensive universities has been the blind area among Erhu education scholars. Based on the researching on some related area and the current Erhu teaching situation at universities, this article proposes some reform measure and it has certain reference value on the current Erhu educating and researching at comprehensive universities. Erhu educating at the comprehensive university should get rid of "the elite education" of the conservatories and avoid the blind copying of the "cultivation of qualified teachers" at the Teachers' Colleges. It should aim at cultivating comprehensive, high quality talents for the society who have strong practice ability and spirit of innovation, who can play and teach Erhu in the theatrical troupe and related teaching organizations.

\section{References}

[1] W. H. Huang, Thinking of Erhu teaching reform in the art colleges, Art Research,3(2006) 58-59

[2] J. J. Yuan, review and research of Erhu textbook in Chinese. Master Degree Thesis Nanjing Normal University, (2006).

[3] Y. G. Xiang,Thinking of Erhu professional teaching in the general undergraduate universities, Journal of Huaihua University, 10(2010)159.

[4] H. Y. Zhao, Erhu primer (Tongxin Publications, China, 2001.

[5] C. F. Liu, Erhu systematic advance gradus(People's Music Publishing House, China, 2002).

[6] G. T. Wang, Erhu scales(People’s Music Publishing House, China, 1987).

[7] G. T. Wang, H. Y. Zhao, Erhu's three hundred basic exercises(People's Music Publishing House, China, 1994).

[8] J. M. Yan, Erhu bowing practice law 37 pieces (People's Music Publishing House, China, 2009)

[9] H. Y. Zhao, Erhu mini practice pieces (People's Music Publishing House, China, 2002).

[10] K. Xue, The style of Erhu gradus (People's Music Publishing House, China, 2010). 
[11] G. T. Wang, H. Y. Zhao, The 158 Erhu style practice pieces (People's Music Publishing House, China, 1994).

[12]H. X. Su, Erhu etudes fluid 26 (ShangHai Music Publishing House, China, 1998). 